\title{
A comparative study among normal saline, water soluble gel and $2 \%$ lidocaine gel as a SLIPA lubricant
}

\author{
Pyung-Gul Park ${ }^{1}$, Geun Joo Choi ${ }^{1}$, Won Joong Kim ${ }^{1}$, So-Young Yang ${ }^{1}$, Hwa-Yong Shin ${ }^{1}$, Hyun Kang ${ }^{1}$, \\ Chong Wha Baek ${ }^{1}$, Yong Hun Jung ${ }^{1}$, Jin-Yun Kim ${ }^{1}$, and Min-Su Kang ${ }^{2}$ \\ Department of Anesthesiology and Pain Medicine, ${ }^{1}$ Chung-Ang University College of Medicne, ${ }^{2}$ National Police Hospital, Seoul, Korea
}

Background: This study was designed to find appropriate lubricant for streamed lined liner of pharyngeal airway ${ }^{\mathrm{TM}}$ $\left(\mathrm{SLIPA}^{\mathrm{TM}}\right)$. We evaluated the incidence of sore throat, nausea, vomiting, hoarseness, paresthesia and blood stain after using saline, water soluble gel and $2 \%$ lidocaine gel as a SLIPA ${ }^{\mathrm{TM}}$ lublicant.

Methods: One hundred twenty three patients scheduled for minor surgery to whom the SLIPA ${ }^{\mathrm{TM}}$ was considered suitable were randomly allocated to one of three groups which receive normal saline, water soluble gel or $2 \%$ lidocaine gel as a SLIPA ${ }^{\mathrm{TM}}$ lublicant. Patients were interviewed at recovery room, post operation 6-12 hour, post operation 18-24 hour about sore throat and other complications.

Results: There were no statistical difference in sore throat and blood stain among three groups. Also there were no statistical differences in hoarseness, nausea, vomiting. The incidence of paresthesia in $2 \%$ lidocaine gel group was significantly higher than those of the other two groups immediately after operation, but it was resolved after leaving the recovery room.

Conclusions: Our results suggest that normal saline, water soluble gel and 2\% lidocaine gel are all available as a SLIPA ${ }^{\mathrm{TM}}$ lubricant. Size of SLIPA ${ }^{\mathrm{TM}}$, insertion technique and difficulty of insertion should be further investigated as the main causes of a sore throat and other complications which can occur after the insertion of SLIPA ${ }^{\mathrm{TM}}$. (Korean J Anesthesiol 2014; 66: 105-111)

Key Words: Lubricant, SLIPA, Sore throat.

Received: March 25, 2013. Revised: 1st, June 3, 2013; 2nd, July 15, 2013; 3rd, August 7, 2013; 4th, August 12, 2013; 5th, August 20 , 2013. Accepted: August 20, 2013.

Corresponding author: Yong Hun Jung, M.D., Ph.D., Department of Anesthesiology and Pain Medicine, Chung-Ang Universtiy College of Medicine, 224-1, Heuksuk-dong, Dongjak-gu, Seoul 156-756, Korea. Tel: 82-2-6299-2571, Fax: 82-2-6299-2585, E-mail: jyh623@cau.ac.kr (c) This is an open-access article distributed under the terms of the Creative Commons Attribution Non-Commercial License (http:// creativecommons.org/licenses/by-nc/3.0/), which permits unrestricted non-commercial use, distribution, and reproduction in any medium, provided the original work is properly cited. 


\section{Introduction}

Compared to an endotracheal tube, the supraglottic airway is less invasive and an easier to learn insertion method, and airway management is easier in difficult situations; thus, its use is increasing in general anesthesia $[1,2]$.

SLIPA $^{\mathrm{TM}}$ (streamed lined liner of pharyngeal airway ${ }^{\mathrm{TM}}$, SLIPA Medical Ltd, London, UK) is a type of supraglottic airway without a cuff, and it is structurally similar to the contour of the pharynx so there is less gas leaks according to the change in head position of patients (Fig. 1). The oropharyngeal leak pressure is also slightly higher than the LMA-ProSeal ${ }^{\mathrm{TM}}$ (laryngeal mask airway ProSeal ${ }^{\mathrm{TM}}$, Laryngeal Mask Company, Berkshire, UK) $[3,4]$. In addition, the SLIPA ${ }^{\mathrm{TM}}$ has a storage space of $50 \mathrm{ml}$, and this is larger than the $25 \mathrm{ml}$ stomach size of a patient who has fasted, so even when regurgitation occurs, this is stored in the storage space and reduces the risk of absorption [5]. There is a report that there was no difference in the occurrence frequency of sore throat, dysphonia, and dysphagia when SLIPA and LMA classic were inserted using water soluble gel by a non-

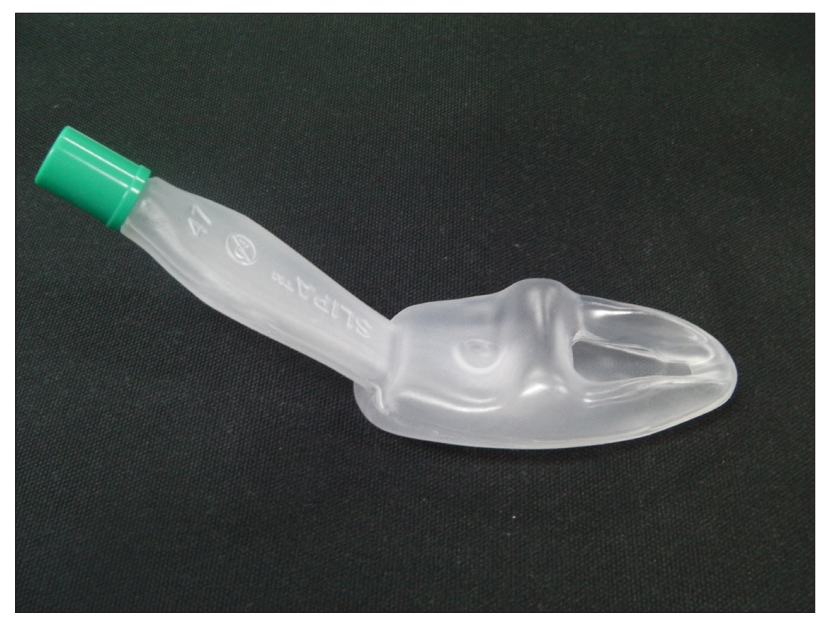

Fig. 1. SLIPA ${ }^{\mathrm{TM}}$ (stream lined liner of pharyngeal airway ${ }^{\mathrm{TM}}$, SLIPA Medical Ltd., London, UK). expert [6]. However, the SLIPA ${ }^{\mathrm{TM}}$ is made of less flexible harder material than LMA-ProSeal ${ }^{\mathrm{TM}}$, so there are reports that there is a higher frequency and severity of sore throat and blood stains on the equipment surface $[4,7]$.

When inserting the LMA-ProSeal ${ }^{\mathrm{TM}}$, the lubricant has a similar function as saliva to help swallowing and prevents sticking to the oropharynx [8]. Normal saline and water soluble gel are recommended as a lubricant for the LMA-ProSeal ${ }^{\mathrm{TM}}$, but there are reports that use of local anesthetic gel can reduce the occurrence of nausea during emergence $[9,10]$, and also that it reduced the frequency of sore throat after endotracheal intubation [11]. However it has also been reported that local anesthetic gel can cause paresthesia and damage the protective reflex integrity in some patients [12]. In addition, the occurrence of nerve damage may not be perceived and it may be difficult to recognize nerve damage caused by an incorrectly mounted SLIPA ${ }^{\mathrm{TM}}$ [13].

Despite the fact that there is a higher occurrence of sore throat and blood stains than that of the LMA-ProSeal ${ }^{\mathrm{TM}}$ due to the material and shape characteristics of SLIPA ${ }^{\mathrm{TM}}$, there is no research regarding the choice of a suitable lubricant. Hence, the authors examined the severity of sore throat after using normal saline, water soluble gel, and $2 \%$ lidocaine gel as lubricants, and compared nausea, vomiting, hoarseness, paresthesia and blood stains after removing SLIPA ${ }^{\mathrm{TM}}$ to find the most suitable lubricant when inserting SLIPA ${ }^{\mathrm{TM}}$.

\section{Materials and Methods}

This research was conducted on 123 patients scheduled for minor surgery, who were American Society of Anesthesiologists physical status classification 1 or 2 and Mallampati class 1 or 2, and between the ages of 20 to 70 . Patients with a history of sore throat within the last 10 days prior to their operation, those with limitations in opening their mouth or extending their neck, a body mass index of 28 or higher, and the risk of aspiration (history of gastrointestinal surgery, esophageal hiatus, and gastrooesophageal reflux) were excluded from the study. There were no differences in age, height, weight, operation time, Mallampati

Table 1. Patient's Demographics

\begin{tabular}{|c|c|c|c|c|}
\hline & $\begin{array}{l}\text { Normal saline } \\
\qquad(\mathrm{n}=43)\end{array}$ & $\begin{array}{l}\text { Water soluble gel } \\
\qquad(\mathrm{n}=41)\end{array}$ & $\begin{array}{l}2 \% \text { lidocaine gel } \\
\qquad(\mathrm{n}=39)\end{array}$ & $P$ value \\
\hline Age (yr) & $49.1 \pm 14.4$ & $45.0 \pm 15.6$ & $45.8 \pm 16.0$ & 0.421 \\
\hline $\operatorname{Sex}(M / F)$ & $19 / 25$ & $19 / 22$ & $20 / 19$ & 0.760 \\
\hline Height $(\mathrm{cm})$ & $158.9 \pm 28.9$ & $164.2 \pm 9.8$ & $165.5 \pm 9.7$ & 0.459 \\
\hline Weight (kg) & $61.1 \pm 9.7$ & $61.0 \pm 12.1$ & $65.0 \pm 11.9$ & 0.239 \\
\hline Inserted time of $\mathrm{SLIPA}^{\mathrm{TM}}(\mathrm{min})$ & $86.0 \pm 45.3$ & $84.5 \pm 37.5$ & $75.0 \pm 35.1$ & 0.386 \\
\hline Mallampati class (I/II) & $25 / 18$ & $25 / 16$ & $24 / 15$ & 0.944 \\
\hline Difficulty of insertion (easy/moderate/diffficult) & $38 / 3 / 2$ & $37 / 2 / 2$ & $35 / 2 / 2$ & 0.996 \\
\hline SLIPA size $(47 / 49 / 51 / 53 / 55 / 57)$ & $4 / 16 / 4 / 14 / 3 / 2$ & $9 / 7 / 7 / 14 / 3 / 1$ & $4 / 9 / 4 / 11 / 8 / 3$ & 0.213 \\
\hline
\end{tabular}

Values are expressed as mean \pm standard deviation or absolute number. There were no significant differences among the groups. 
class, and difficulty of insertion between the groups (Table 1). The protocol for this research was approved by the Institutional Board of University, and the research was conducted according to the principles of the 2010 Declaration of Helsinki. Written consent was obtained from all participants before the research and the type of lubricant used was not revealed.

According to the type of lubricant, the subjects were divided into 3 groups: normal saline (sodium chloride ${ }^{\mathrm{TM}}$, Huons, Jecheon, Korea), water soluble gel (chlorhexidine cream $^{\mathrm{TM}}$, Alico, Jecheon, Korea), and 2\% lidocaine gel (lidocaine HCL Jelly $2 \%{ }^{\mathrm{TM}}$, Alico, Jecheon, Korea). A random number generator program in Microsoft Excel was used for the randomization. The subjects were allocated into groups by giving a sealed envelope with a number inside to an inspector, and after the patient entered the operating room and directly before inducing anesthesia, the envelope was opened to find out which group the patient was allocated to (Fig. 2).

As premedication before surgery, glycopyrrolate $0.2 \mathrm{mg}$ was IV injected in the ward an hour before anesthesia induction. After arriving in the operating room, electrocardiogram, pulse oximetry, end-tidal $\mathrm{PCO}_{2}$, and non-invasive blood pressure were monitored. Three minutes before anesthesia induction, preoxygenation was performed. Then, fentanyl $2 \mu \mathrm{g} / \mathrm{kg}$ was IV injected, and 1 minute later, thiopental sodium $5 \mathrm{mg} / \mathrm{kg}$ was administered. The lubricant was thinly applied to the back side of the SLIPA ${ }^{\mathrm{TM}}$ before insertion and care was taken so it would not dry out. When the jaw was sufficiently relaxed after losing the eyelash reflex and administering rocuronium $0.6 \mathrm{mg} / \mathrm{kg}$, the SLIPA ${ }^{\mathrm{TM}}$ was inserted with the standardized method recommended by the product's company. After extending the head and bending the neck (sniffing position), sterile gloves were worn and a thumb held the inside the mouth and an index finger held the chin to lift it up. SLIPA ${ }^{\mathrm{TM}}$ was pushed into the neck toward the esophagus following its curvature, and the heel area was positioned to reach the nasopharynx region. The size of the SLIPA $^{\mathrm{TM}}$ was selected according to the standards provided by the product's company set according to the patient's height. The insertion of SLIPA ${ }^{\mathrm{TM}}$ was performed by a single practitioner who has used the LMA-ProSeal ${ }^{\mathrm{TM}}$ for 3 years and had performed SLIPA $^{\mathrm{TM}}$ insertion 60 times or more. The difficulty of insertion was also subjectively evaluated by the same single practitioner. When the insertion of SLIPA ${ }^{\mathrm{TM}}$ was not successful on the first attempt, it was considered as a failed attempt. A single attempt was made to position the SLIPA ${ }^{\mathrm{TM}}$ inside the oral cavity and to remove it from the oral cavity. Successful insertion was considered as when the maximum oropharyngeal leak pressure exceeded 15 $\mathrm{cmH}_{2} \mathrm{O}$ (where the figure of the pressure gauge did not increase further during manually assisted ventilation), the movement of the chest wall was normal during mechanical ventilation, and end-tidal $\mathrm{PCO}_{2}$ appeared as a normal waveform. Anesthesia was maintained with nitrous oxide $1.5 \mathrm{~L} / \mathrm{min}$, oxygen $1.5 \mathrm{~L} / \mathrm{min}$, and $1.5-2.5$ vol\% sevoflurane. The same anesthesia machine was used during this research (Aestiva/5, Datex-Ohmeda Inc, Madison, USA), and humidifying equipment was not used. The tidal volume was controlled as $8-10 \mathrm{ml} / \mathrm{kg}$, and maximum respiratory pressure was controlled to not exceed $17 \mathrm{cmH}_{2} \mathrm{O}$. Inhalation and expiration was $1: 2$, and the breathing rate was set to maintain the end-tidal $\mathrm{PCO}_{2}$ within the normal range $(35-40 \mathrm{mmHg})$. While regaining consciousness, the SLIPA ${ }^{\mathrm{TM}}$ was removed after confirming that sufficient spontaneous respiration had been restored, and the equipment was checked for blood stains. The settings for patient controlled analgesia followed the standard-

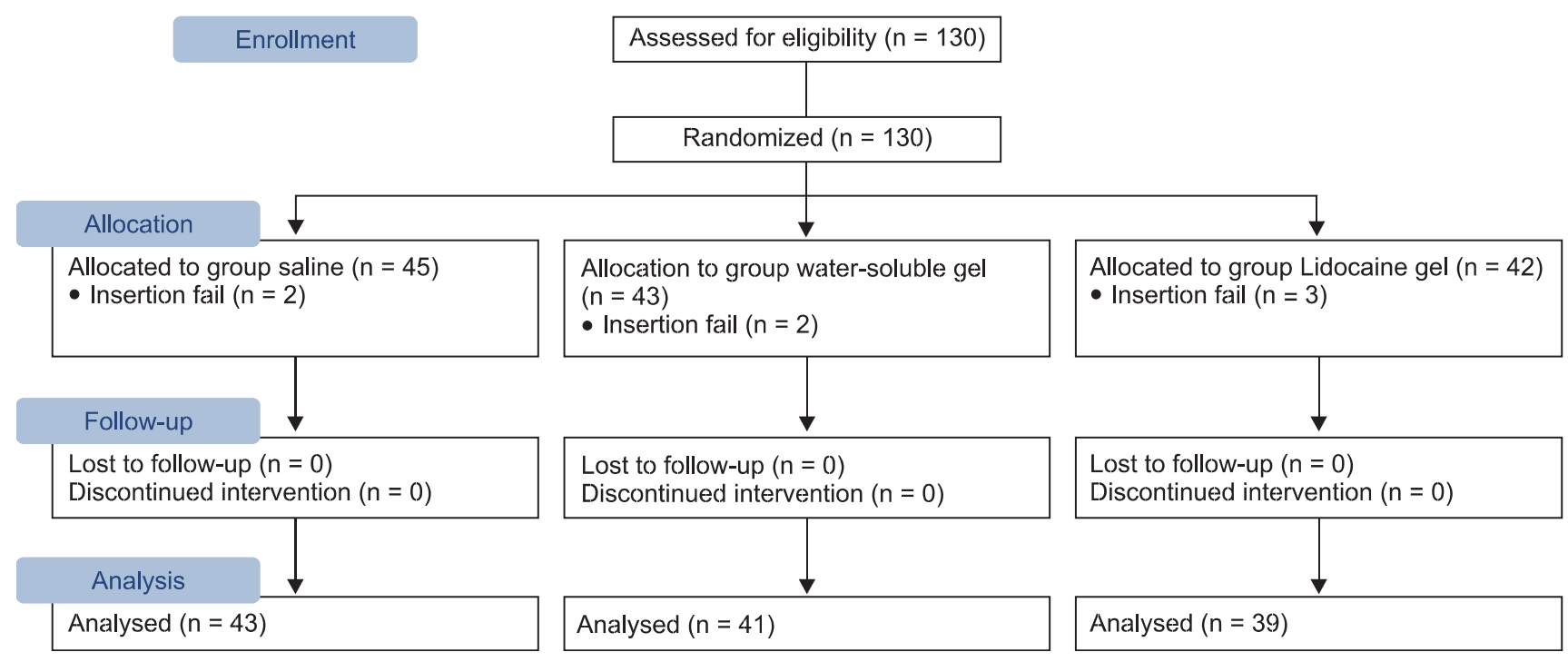

Fig. 2. Consort flow chart. 
ized method of the hospital according to operation type, age, and weight, and it was administered in the recovery room after checking for sore throat on a numeric rating scale (NRS 0: no pain - NRS 10: unimaginable maximum pain). Fentanyl $50 \mu \mathrm{g}$ was used as postoperative analgesic to control pain when the numeric rating scale was 3 or more.

After the patients were fully awake, an anesthesiologist unaware of the lubricant type interviewed the patients at 6-12 hours after the operation and 18-24 hours after the operation. Each patient was questioned regarding postoperative discomfort, pain, paresthesia, hoarseness, nausea, and vomiting, and the degree of sore throat was scored using a numeric rating scale. Cases where the insertion of SLIPA ${ }^{\mathrm{TM}}$ had been attempted 2 or more times were excluded from the analysis. To check whether the protective reflex integrity of the respiratory tract was lost, the patients were observed until complete consciousness for aspiration.

The primary end point of this research was the degree of sore throat evaluated with the numeric rating scale. A pilot study was conducted on 10 patients receiving minor surgery for each group using normal saline, water soluble gel, and $2 \%$ lidocaine gel during the insertion of SLIPA ${ }^{\mathrm{TM}}$, and the mean and standard deviation of the numeric rating scale was $2.1 \pm 1.4,1.8 \pm 1.1$, and $1.3 \pm 1.2$. When a $5 \%$ error and $80 \%$ test power were applied, the required number of subjects was calculated to be 37 patients for each group. Here, a compliance rate of $90 \%$ and an insertion failure rate of $5 \%$ were applied and a total of 130 patients were required.

To compare the groups, for continuous data, the SharpiroWilk test was used to evaluate normality. Data showing normal distribution were expressed as the mean \pm standard deviation,

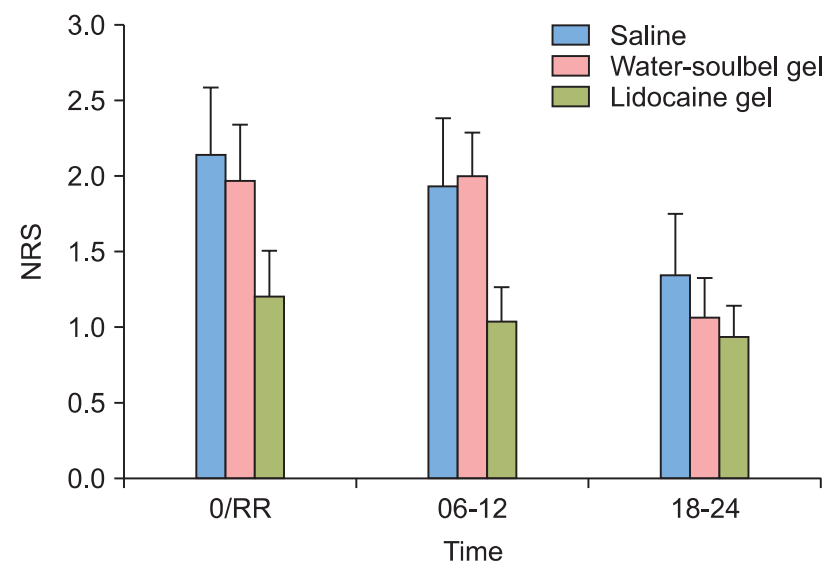

Fig. 3. Sore throat at three time zones in each group. 0/RR: at recovery room, 6-12: post operation 6-12 hour, 18-24: post operation 18-24 hour. This graph is changes of sore throat according to the type of lubricant. There were no significant differences among the groups. Values are expressed as mean \pm standard error (SE). NRS: numeric rating scale. and each group was compared with variance analysis and Tukey test as a post test. Results showing a non-normal distribution were expressed as the median value (interquartile range), and were analyzed using the Kruskal-Wallis test and Bonferroni correction. The change in numeric rating scale according to the group and time was analyzed using repeated measure ANOVA.

Discrete variables were presented as a number (\%), and were analyzed using $\mathrm{x}^{2}$ analysis or Fischer's exact test. Results were considered statistically significant when the $\mathrm{P}$ value was $<0.05$. All analyses were performed using SPSS 18.0 (IBM Co., Armonk, NY, USA).

\section{Results}

According to the type of lubricant, the subjects were divided into three groups: a normal saline group, water soluble gel group, and $2 \%$ lidocaine group (Table 1 ).

In comparing lubricants for the insertion of SLIPA ${ }^{\mathrm{TM}}$, sore throat had a lower numeric rating scale for all time periods of complete awakening after operation, 6-12 hours after operation, and 18-24 hours after operation, but there was no statistically significant difference between the three groups (Fig. 3). There was also no statistical difference in the number of analgesic administrations (Table 2).

There was no difference between the three groups in blood stains on the equipment after removing SLIPA ${ }^{\mathrm{TM}}$, and the incidence of hoarseness, nausea, and vomiting was also not different between the three groups at complete awakening after operation, 6-12 hours after operation, and 18-24 hours after operation. Occurrence of paresthesia in the oral cavity or laryngopharynx was not different between the normal saline group and water soluble gel group, but it occurred at a significantly higher level in the $2 \%$ lidocaine group compared to the other groups, and there was no loss of protective reflex integrity which represses aspiration in all patients (Table 2).

\section{Discussion}

This research examined the correlation between sore throat and the type of lubricant used for SLIPA ${ }^{\mathrm{TM}}$ as the primary end point. There was no difference in sore throat between the three groups. There was also no difference in blood stains, hoarseness, nausea, and vomiting between the groups, but paresthesia occurred significantly more in the $2 \%$ lidocaine group.

The incidence of sore throat caused by the LMA-ProSeal ${ }^{\mathrm{TM}}$ has been diversely reported at $0-50 \%$, but mostly it has been reported to be around $10 \%$. In most cases, it is not severe, and it is lower than the incidence caused by endotracheal intubation and similar to the incidence caused by face mask [14]. It is not clear why the incidence reported for sore throat is so diverse, but it is 
Table 2. Complications of SLIPA ${ }^{\mathrm{TM}}$

\begin{tabular}{|c|c|c|c|c|}
\hline & $\begin{array}{l}\text { Normal saline } \\
\quad(\mathrm{n}=43)\end{array}$ & $\begin{array}{l}\text { Water soluble gel } \\
\qquad(\mathrm{n}=41)\end{array}$ & $\begin{array}{l}2 \% \text { lidocaine gel } \\
\quad(\mathrm{n}=39)\end{array}$ & P value \\
\hline Blood stain & $3(7.0)$ & $2(4.9)$ & $2(5.1)$ & 0.915 \\
\hline \multicolumn{5}{|l|}{ Hoarseness } \\
\hline $\mathrm{RR}$ & $6(13.6)$ & $5(12.2)$ & $4(10.3)$ & 0.895 \\
\hline PO 6-12 & $5(11.4)$ & $5(12.2)$ & $3(7.7)$ & 0.783 \\
\hline PO $18-24$ & $4(9.1)$ & $1(2.4)$ & $3(7.7)$ & 0.427 \\
\hline \multicolumn{5}{|l|}{ Nausea } \\
\hline $\mathrm{RR}$ & $8(18.2)$ & $5(12.2)$ & $5(12.8)$ & 0.689 \\
\hline PO 6-12 & $6(13.6)$ & $3(7.3)$ & $3(7.7)$ & 0.542 \\
\hline PO $18-24$ & $2(4.5)$ & $1(2.4)$ & $1(2.6)$ & 0.826 \\
\hline \multicolumn{5}{|l|}{ Vomiting } \\
\hline $\mathrm{RR}$ & $5(11.4)$ & $2(4.9)$ & $1(2.6)$ & 0.234 \\
\hline PO 6-12 & $2(4.5)$ & $2(4.9)$ & $2(5.1)$ & 0.992 \\
\hline PO $18-24$ & $0(0)$ & $0(0)$ & $0(0)$ & NA \\
\hline \multicolumn{5}{|l|}{ Paresthesia } \\
\hline $\mathrm{RR}$ & $1(2.3)$ & $4(9.8)$ & $15(38.5)^{*, \dagger}$ & $<0.001$ \\
\hline PO 6-12 & $0(0)$ & $1(2.4)$ & $2(5.1)$ & 0.316 \\
\hline PO $18-24$ & $0(0)$ & $0(0)$ & $0(0)$ & NA \\
\hline Protective reflex integrity & $0(0)$ & $0(0)$ & $0(0)$ & NA \\
\hline Additional analgesic administration & $4(9.3)$ & $3(7.3)$ & $3(7.7)$ & 0.939 \\
\hline
\end{tabular}

RR: at recovery room, PO 6-12: post operation 6-12 hour, PO 18-24: post operation 18-24 hour, NA: not applicable. Values are expressed as absolute number $(\%) . * \mathrm{P}<0.05$ compared with normal saline group, ${ }^{\dagger} \mathrm{P}<0.05$ compared water soluble gel group.

considered to be from various latent factors such as the insertion technique, pressure applied to the laryngopharynx membrane, operation time, humidification, and type of lubrication. There is a report that the incidence and severity of sore throat can be reduced when the pressure of the cuff is decreased in the LMAProSeal $^{\mathrm{TM}}$ [15]. In addition, in a study on the Cobra PLA ${ }^{\mathrm{TM}}$ (Cobra perilaryngeal airway ${ }^{\mathrm{TM}}$, Engineered Medical Systems, Indianapolis, USA), the incidence and severity of sore throat decreased when the cuff pressure was reduced [16]. I-gel ${ }^{\mathrm{TM}}$ is a type of supraglottic airway with no cuff similarly to SLIPA ${ }^{\mathrm{TM}}$ and there is a report that $\mathrm{i}-$ gel $^{\mathrm{TM}}$ (Intersurgical Ltd., Wokingham, Berkshire, UK) had no difference in the incidence and severity of sore throat compared to existing LMA [17]. This is considered to be because the i-gel ${ }^{\mathrm{TM}}$ is made of a flexible material unlike the SLIPA $^{\mathrm{TM}}$.

In this study, there was no statistical difference in sore throat between the three groups according to the type of lubricant. In a study that compared normal saline and $2 \%$ lidocaine gel as a lubricant in the use of the LMA-ProSeal ${ }^{\mathrm{TM}}$, there was also no difference in sore throat between the two groups [18]. From our research and precedent research regarding supraglottic airway, the incidence of sore throat when using SLIPA ${ }^{\mathrm{TM}}$ is not significantly influenced by the type of lubricant, but instead it is considered that a significant difference will be seen according to the pressure applied on the mucous membrane. The pressure on the mucous membrane cannot be controlled through the cuff in the case of SLIPA $^{\mathrm{TM}}$, and the size of the SLIPA ${ }^{\mathrm{TM}}$ on the laryngopharynx can act as an important factor due to its hard material.
In this study, the size was selected according to the standards set for height provided by the product's manufacturer, but there is need for further research regarding the suitable size for patients such as selecting a SLIPA ${ }^{\mathrm{TM}}$ size according to the width of the thyroid cartilage. Also, additional analgesic was used when the numeric rating scale was 3 or higher, and 4 patients from the normal saline group, 3 patients from the water soluble gel group, and 3 patients from the $2 \%$ lidocaine gel group used additional analgesic. However, there were instances where it was administered for pain in the operated area, and the spatial parameters of the analgesic administration time and interview time were limitations in this research.

There was no difference in the existence of blood stains on the equipment after removing the SLIPA ${ }^{\mathrm{TM}}$ between the three groups. A total of 13 patients showed a difficulty of medium or higher although the SLIPA ${ }^{\mathrm{TM}}$ was inserted in the first attempt, and from these, 5 patients showed blood stains. For the LMAProSeal $^{\mathrm{TM}}$, there is a study reporting that the frequency of blood stains was higher when the insertion was attempted several times [18]. From the above results, the insertion difficulty and suitable size of the inserting body are considered to be influential in the occurrence of blood stains.

Occurrence of hoarseness was not different between the three groups according to the type of lubricant used with the SLIPA $^{\mathrm{TM}}$. In a study that compared normal saline and $2 \%$ lidocaine gel with the LMA-ProSeal $^{\mathrm{TM}}$, hoarseness occurred only in 4 patients in the lidocaine gel group, and from these, insertion was attempted 2 or more times in 2 patients [18]. Occurrence of 
hoarseness is also considered to be mainly influenced by the insertion difficulty and suitable size of the inserting body, besides the type of lubricant $[3,18]$.

Occurrence of paresthesia in the oral cavity and laryngopharynx was significantly higher in the $2 \%$ lidocaine group compared to the other two groups. Patients who complained of paresthesia mostly complained of foreign body sensation or numbness in the oral cavity and laryngopharynx, and it did not cause loss of protective reflex integrity which represses aspiration.

For the LMA-ProSeal ${ }^{\mathrm{TM}}$, use of local lidocaine has been reported to minimize nausea and vomiting during awakening. Chan et al. reported that the use of lidocaine gel in a short procedure reduced nausea from 47 to 17\% [9]. In addition, Millet and Alleman [10] reported that the use of $10 \%$ lidocaine spray before inducing anesthesia had the effect of reducing nausea from the procedure to within 30 minutes. However, regardless of cuff pressure control or use of anesthetic lubricant with the LMAProSeal $^{\mathrm{TM}}$, when the practitioner is skilled at using the LMAProSeal $^{\mathrm{TM}}$, the occurrence of nausea has been reported to be less than $2 \%$ [19]. In addition, when the cuff pressure of the LMAProSeal $^{\mathrm{TM}}$ was controlled, nausea did not occur regardless of the use of lidocaine gel. In a study which compared normal saline and $2 \%$ lidocaine gel as lubricants with the LMA-ProSeal ${ }^{\mathrm{TM}}$, the incidence of nausea was not different between the two groups [18]. Additionally, in our study, there was no difference in the incidence of nausea between the three groups which used normal saline, water soluble gel, and 2\% lidocaine gel as lubricants for SLIPA ${ }^{\mathrm{TM}}$. The incidence of nausea from the use of SLIPA ${ }^{\mathrm{TM}}$ does not seem to be significantly influenced by the type of lubricant, but as some LMA-ProSeal ${ }^{\mathrm{TM}}$ related studies mentioned above have reported, there is an effect from the lubricant so further research is necessary.

There was no difference in the incidence of sore throat, blood stains, hoarseness, nausea, and vomiting between the three lubricants. Occurrence of paresthesia was significantly higher in the $2 \%$ lidocaine gel group, but it did not induce the loss of protective reflex integrity which represses absorption. Therefore, normal saline, water soluble gel, and $2 \%$ lidocaine gel can all be used as lubricants for SLIPA ${ }^{\mathrm{TM}}$. In addition, as mentioned above, the suitable size of SLIPA ${ }^{\mathrm{TM}}$ and insertion difficulty are considered to be a major factor for causing a sore throat and other complications, so there is need for further study regarding this area $[3,18]$.

\section{Acknowledgments}

This research was supported by Basic Science Research Program through the National Research Foundation of Korea (NRF) funded by the Ministry of Education, Science and Technology (2012R1A1A1003700).

\section{References}

1. Robak O, Leonardelli M, Zedtwitz-Liebenstein K, Rützler K, Schuster E, Vaida S, et al. Feasibility and speed of insertion of seven supraglottic airway devices under simulated airway conditions. CJEM 2012; 14: 330-4.

2. Timmermann A. Supraglottic airways in difficult airway management: successes, failures, use and misuse. Anaesthesia 2011; 66 Suppl 2: 4556.

3. Woo YC, Cha SM, Kang H, Baek CW, Jung YH, Kim JY, et al. Less perilaryngeal gas leakage with SLIPA ${ }^{\mathrm{TM}}$ than with LMA-ProSeal ${ }^{\mathrm{TM}}$ in paralyzed patients. Can J Anaesth 2011; 58; 48-54.

4. Abdellatif AA, Ali MA. Comparison of streamlined liner of the pharynx airway $\left(\mathrm{SLIPA}^{\mathrm{TM}}\right)$ with the laryngeal mask airway Proseal ${ }^{\mathrm{TM}}$ for lower abdominal laparoscopic surgeries in paralyzed, anesthetized patients. Saudi J Anaesth 2011; 5: 270-6.

5. Miller DM, Light D. Laboratory and clinical comparisons of the Streamlined Liner of the Pharynx Airway (SLIPA) with the laryngeal mask airway. Anaesthesia 2003; 58: 136-42.

6. Oh SK, Lim BG, Kim H, Lim SH. Comparison of the clinical effectiveness between the streamlined liner of pharyngeal airway (SLIPA) and the laryngeal mask airway by novice personnel. Korean J Anesthesiol 2012; 63: 136-41.

7. Choi YM, Cha SM, Kang H, Baek CW, Jung YH, Woo YC, et al. The clinical effectiveness of the streamlined liner of pharyngeal airway (SLIPA) compared with the laryngeal mask airway ProSeal during general anesthesia. Korean J Anesthesiol 2010; 58: 450-7.

8. Brain AI. Modification of laryngeal mask insertion technique in children. Anesth Analg 1995; 81: 212.

9. Chan ST, Tham CS. The effects of $2 \%$ lignocaine gel on incidence of retching with the use of the laryngeal mask airway. Anaesthesia 1995; 50: 257-8.

10. Millett SV, Alleman KG. Lignocaine gel and the laryngeal mask airway. Anaesthesia 1995; 50: 747.

11. Sumathi PA, Shenoy T, Ambareesha M, Krishna HM. Controlled comparison between betamethasone gel and lidocaine jelly applied over tracheal tube to reduce postoperative sore throat, cough, and hoarseness of voice. Br J Anaesth 2008; 100: 215-8.

12. Edwards ND. Lignocaine gel and the laryngeal mask airway. Anaesthesia 1995; 50: 746-7.

13. Inomata S, Nishikawa T, Suga A, Yamashita S. Transient bilateral vocal cord paralysis after insertion of a laryngeal mask airway. Anesthesiology 1995; 82: 787-8. 
14. Brimacombe J, Berry A. Insertion of the laryngeal mask airway-a prospective study of four techniques. Anaesth Intensive Care 1993; 21: 8992.

15. Burgard G, Möllhoff T, Prien T. The effect of laryngeal mask cuff pressure on postoperative sore throat incidence. J Clin Anesth 1996; 8: 198201.

16. Joe HB, Kim DH, Chae YJ, Kim JY, Kang M, Park KS. The effect of cuff pressure on postoperative sore throat after Cobra perilaryngeal airway. J Anesth 2012; 26: 225-9.

17. Das B, Mitra S, Jamil SN, Varshney RK. Comparison of three supraglottic devices in anesthetised paralyzed children undergoing elective surgery. Saudi J Anaesth 2012; 6: 224-8.

18. Keller C, Sparr HJ, Brimacombe JR. Laryngeal mask lubrication. A comparative study of saline versus $2 \%$ lignocaine gel with cuff pressure control. Anaesthesia 1997; 52: 592-7.

19. Brimacombe J. Analysis of 1500 laryngeal mask uses by one anaesthetist in adults undergoing routine anaesthesia. Anaesthesia 1996; 51: 7680. 